\title{
Methods of weaning preterm babies $<30$ weeks gestation off CPAP: a multicentre randomised controlled trial
}

\author{
David A Todd, ${ }^{1}$ A Wright, ${ }^{2}$ M Broom, 1 M Chauhan, \\ S Meskell, ${ }^{1}$ C Cameron, ${ }^{2}$ A M Perdomi, ${ }^{2}$ M Rochefort, ${ }^{2}$ \\ L Jardine, ${ }^{3}$ A Stewart, ${ }^{4}$ B Shadbolt ${ }^{5}$
}

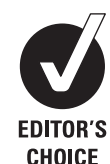

ABSTRACT

Background Controversy exists whether different continuous positive airway pressure (CPAP) weaning methods influence time to wean off CPAP, CPAP duration, oxygen duration, Bronchopulmonary Dysplasia (BPD) or length of admission.

Aims In a multicentre randomised controlled trial, the authors have primarily compared CPAP weaning methods impact on time to wean off CPAP and CPAP duration and secondarily their effect on oxygen duration, BPD and time of admission.

Methods Between April 2006 and October 2009, 177 infants $<30$ weeks gestational age (GA) who fulfilled stability criteria on CPAP were randomised to one of the three CPAP weaning methods (M).

M1: Taken 'OFF' CPAP with the view to stay 'OFF'. M2: Cycled on and off CPAP with incremental time 'OFF'.

M3: As with M2, cycled on and off CPAP but during 'OFF' periods were supported by $2 \mathrm{~mm}$ nasal cannula at a flow of $0.5 \mathrm{l} / \mathrm{min}$.

Results Based on intention to treat analysis, there was no significant difference in mean $\mathrm{GA}$ or birthweight between the groups $(27.1 \pm 1.4$, $26.9 \pm 1.6$ and $27.3 \pm 1.5$ (weeks $\pm 1 S D$ ) and $988 \pm 247,987 \pm 249$ and $1015 \pm 257$ (grams $\pm 1 S D$ ), respectively). Primary outcomes showed M1 produced a significantly shorter

1Centre for Newborn Care (CNC), Canberra Hospital \& Australian National University Medical School, Canberra, Australia

2CNC, Westmead Hospital, Westmead, Australia 3Department of Neonatology, Mater Mothers' Hospital, Brisbane, Australia

4Grantley Stable Neonatal intensive care unit, Royal Brisbane \& Women's Hospital, Brisbane, Australia 5Department of Epidemiology, Canberra Hospital, Canberra, Australia

Correspondence to Dr David A Todd, Department of Neonatology, Canberra Hospital, P0 Box 11, Woden Canberra 2606, Australia; David.Todd@act.gov.au time to wean from CPAP $(11.3 \pm 0.8,16.8 \pm 1.0$, $19.4 \pm 1.3$ (days $\pm 1 S E) p<0.0001$, respectively) and CPAP duration $(24.4 \pm 0.1,38.6 \pm 0.1$,

$30.5 \pm 0.1$ (days $\pm 1 S E) p<0.0001$, respectively). All the secondary outcomes were significantly shorter with $\mathrm{M1}$, loxygen duration: $24.1 \pm 1.5$, $45.8 \pm 2.2,34.1 \pm 2.0$ (days $\pm 1 S E$ ) $p<0.0001$, BPD: 7/56 (12.5\%), 29/69 (42\%), 10/52 (19\%) $p=0.011$ and length of admission: $58.5 \pm 0.1,73.8 \pm 0.1$ $69.5 \pm 0.1$ (days $\pm 1 S E) p<0.0001$, respectively). Conclusion Method 1 significantly shortens CPAP weaning time, CPAP duration, oxygen duration, BPD and admission time.

\section{INTRODUCTION}

Continuous positive airway pressure (CPAP) has been used in preterm babies as a mode of respiratory support since the 1970s and is now used in most NICUs. ${ }^{1-5}$ Subsequently, it has been shown that CPAP may reduce the need for invasive intubation and ventilation, reduce apnoea of prematurity and postextubation atelectasis. Early use of CPAP reduces the incidence of Bronchopulmonary Dysplasia (BPD (defined as an oxygen requirement at 36 weeks corrected gestational age)) and the need for home oxygen. ${ }^{5-11}$

There are several ways of delivering CPAP including head chamber, facemask, nasal prongs and endotracheal tubes. ${ }^{5-8}$ Research and clinical experience have shown that nasal CPAP with nasal prongs is the most efficient way of delivering continuous distending pressure (CDP) to the alveoli. ${ }^{5-8}$ Once infants are stabilised and breathing adequately on CPAP, the CPAP is usually weaned off gradually. ${ }^{6}$

7 Controversy continues over the best method of weaning CPAP and is often approached in an 'ad hoc' manner. 57 12-14

Four trials have compared methods of weaning CPAP and its impact on CPAP

\section{What we already know}

1. Most premature infants require nasal CPAP as a primary or secondary treatment.

2. Criteria when to wean off CPAP and when to reinstate CPAP have not been established.

3. There is very little evidence on the best methods of weaning babies off CPAP.

\section{What this study adds}

1. Using the set stability and failure criteria of the study, taking babies off CPAP with the intention of discontinuing support with CPAP reduces the following:

- Length of weaning time and time on CPAP

- Oxygen duration and BPD

- Duration of stay in the neonatal intensive care unit

duration. ${ }^{15-18}$ The first trial studied the changes in respiratory parameters in infants $<34$ weeks gestational age (GA) requiring CPAP. 15 Once stable in air, they were taken OFF CPAP for $6 \mathrm{~h}$, the trial results found a reduced need for CPAP in these infants. ${ }^{15}$ Two of the four trials have shown weaning CPAP is better achieved by slowly decreasing the CPAP pressure rather than cycling the time off without reducing pressure. ${ }^{16}$ 17 In the fourth trial, results showed in infants $>27$ weeks GA transferring from CPAP to nasal cannula at a flow of $21 / \mathrm{min}$ and oxygen requirement $<31 \%$ made no difference in success to wean from CPAP but increased the time in oxygen and duration of respiratory support. ${ }^{18}$ To date no trials have compared different methods of weaning CPAP OFF preterm infants $<30$ weeks GA at lower oxygen concentrations who are stable on lower CPAP pressures.

\section{AIMS}

The primary aims were to determine whether different CPAP weaning methods affect (1) time to wean off CPAP and (2) CPAP duration.

The secondary aims were to determine whether different CPAP weaning methods affect (1) oxygen duration and $\mathrm{BPD}$ (defined as requiring oxygen at 36 weeks corrected GA to maintain oxygen saturations $>86 \%$ ) and (2) length of admission. 
Stability criteria (must have all 8 criteria for $\geq 12$ hours):-

1. CPAP $4-6 \mathrm{~cm} \mathrm{H}_{2} \mathrm{O}$

2. Oxygen requirement less than $25 \%$ and not increasing

3. Respiratory rate less than 60

4. No significant chest recession (sternal/diaphragmatic)

5. Less than 3 episodes of self reverting apnoeas ( $<20$ seconds) and/or bradycardias (<100 BPM) and/or desaturations $(\leq 86 \%)$ in 1 hour for the previous 6 hours

6. Average saturation $>86 \%$ most of the time or $\mathrm{PaO}_{2} /$ transcutaneous $\mathrm{PaO}_{2}>45 \mathrm{~mm} \mathrm{Hg}$

7. Not currently treated for patent ductus arteriosus or sepsis

8. Tolerated time off CPAP during cares (up to 15 minutes)

Figure 1 Stability criteria.

\section{Criteria for failed trial "OFF" (at least 2 of the following):-}

1. Increase work of breathing (Intercostal recession and accessory muscles contributing for respiration) with respiratory rate $>75$.

2. Increased apnoea and/or bradycardia and/or desaturations $>2$ in 1 hour for the previous 6 hour period.

3. Increased $\mathrm{O}_{2}$ requirement $>25 \%$ to maintain the oxygen saturations $>86 \%$ and/or $\mathrm{PaO}_{2} /$ transcutaneous $\mathrm{PaO}_{2}>45 \mathrm{~mm} \mathrm{Hg}$.

4. $\mathrm{pH}$ of $<7.2$

5. $\mathrm{PaCO}_{2}$ /transcutaneous $\mathrm{PaCO}_{2}>65 \mathrm{mmHg}$.

6. Major apnoea or bradycardia requiring resuscitation

Figure 2 Criteria for failed trial off CPAP.

\section{METHODS}

The CPAP weaning trial was registered with the Australian trials network (Perinatal Trials registration number ACTRN012606000155594 (registered 20/4/2006)) and was approved by each of the Hospitals Research Ethics Committee.
A Data Monitoring Committee (DMC) oversaw the conduct and results of the trial. The trial sites included (1) Canberra Hospital (Co-ordinating centre) which commenced recruiting in January 2007 (2) Westmead Hospital (Sydney) which commenced recruiting in April 2006 (3) the Royal Brisbane and Women's Hospital which commenced recruiting in July 2008. Between April 2006 and October 2009, all premature infants $<30$ weeks GA who were treated with CPAP from birth or required nasal CPAP secondarily following extubation were considered for inclusion in this randomised controlled trial (RCT).

Enrolled premature infants were randomly allocated to one of the three methods of weaning CPAP. Recruitment was completed with prior consent from the parents and occurred once the infants were approaching the 'stability criteria' (figure 1). Exclusion criteria included the following: (1) CPAP for <24 h; (2) parents refused consent or weaning already commenced without consent; (3) need for surgery; (4) transferred to other hospital; (5) oxygen requirements $>25 \%$; (6) infant $<750 \mathrm{~g}$ at time of randomisation; (7) congenital abnormalities; (8) > grade 2 intraventricular haemorrhage (IVH); (9) subsequently died.

Routine clinical management was provided for all premature infants $<30$ weeks GA with CPAP pressure and oxygen requirement adjusted as directed by the medical team. Only once the babies were stable on a CPAP pressure of 4-6 $\mathrm{cmH} 2 \mathrm{O}$ for $\geq 24 \mathrm{~h}$ and they had satisfied the 'stability criteria' were babies randomised to a weaning method.

Babies were stratified by trial site and GA (<28 weeks vs 28-29 weeks) and a ratio of 1:1:1 was used by DiscoverQuick's artificially intelligent randomisation engine to allocate premature infants to the weaning method within the strata. The trial was initially started at one site using a manual 'simple' randomisation approach (sealed envelopes) but this site converted to the web-based method once the second hospital began recruiting.

The three methods were as follows:

- Method 1 (M1): CPAP was taken 'OFF' and the premature infant remained in crib oxygen or air with a view to stay off CPAP. If the infant failed without CPAP support (figure 2: 'failure criteria'), they were recommenced 'ON' CPAP at the level of CPAP when taken 'OFF' for at least $48 \mathrm{~h}$ and the infant did not trial off again until the stability criteria was again satisfied.

- Method 2 (M2): CPAP was weaned gradually by cycling between intervals of periods of time 'OFF' followed by a fixed period of $6 \mathrm{~h}$ ' $O \mathrm{~N}$ ' CPAP. When 'OFF' CPAP, the baby remained in crib oxygen or air as required. The period of time 'OFF' was gradually increased $(2-4 \mathrm{~h}$ 


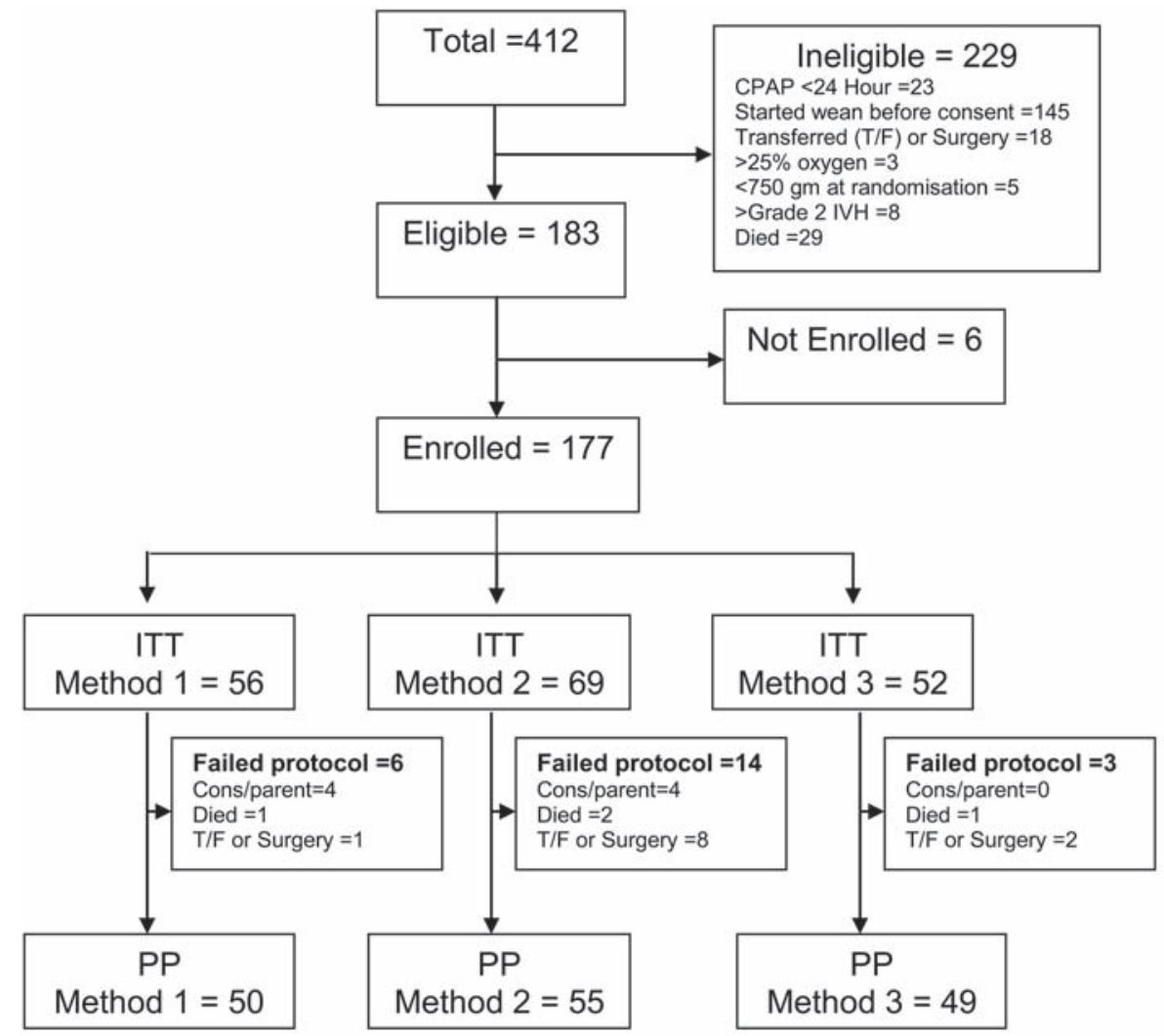

Figure 3 Total preterm babies, those eligible and enrolled in the trial (intention to treat (ITT) and per protocol (PP)).

Table 1 Baseline characteristics by CPAP weaning method $(n=177)$

\begin{tabular}{lllll}
\hline & $\mathrm{M} 1(\mathrm{n}=56)$ & $\mathrm{M} 2(\mathrm{n}=69)$ & $\mathrm{M} 3(\mathrm{n}=52)$ & $\mathrm{Sig}$ \\
\hline GA (weeks) & $27.1 \pm 1.4 \dagger$ & $26.9 \pm 1.6$ & $27.3 \pm 1.5$ & $\mathrm{NS}$ \\
BWt (g) & $988 \pm 247 \dagger$ & $987 \pm 249$ & $1015 \pm 257$ & $\mathrm{NS}$ \\
$\mathrm{F}: \mathrm{M}(\mathrm{M} \%)$ & $37: 19(34 \%)$ & $27: 42(64 \%)$ & $25: 27(52 \%)$ & $\mathrm{p}=0.01$ \\
Apgar 1 & $5(3-6) \ddagger$ & $4(3-6)$ & $6(4-7)^{*}$ & $\mathrm{p}=0.006$ \\
Apgar 5 & $7(6-8) \ddagger$ & $7(6-8)$ & $8(7-9)^{*}$ & $\mathrm{p}=0.01$ \\
Surfactant (Y/N) & $53: 3(95 \%)$ & $61: 8(88 \%)$ & $46: 6(88 \%)$ & $\mathrm{NS}$ \\
CGA start wean (weeks) & $29.6 \pm 1.7 \dagger$ & $30.5 \pm 2.3$ & $29.7 \pm 1.9$ & $\mathrm{NS}$ \\
CWt start & $1014 \pm 38 \dagger$ & $1112 \pm 34$ & $1074 \pm 40$ & $\mathrm{NS}$ \\
wean (g) & & & & \\
\hline
\end{tabular}

*Significantly higher than M1 \& M2 p $<0.01$.

tMean \pm 1 SD

$¥$ Median (range)

$\mathrm{BWt}$, birthweight; CGA, corrected GA; CWt, corrected Wt; GA, gestational age.

increase). Once the baby was able to tolerate $16 \mathrm{~h}$ 'OFF' CPAP an attempt was made to stop CPAP at the next period off. If the infant met the 'failure criteria' the baby was re-commenced 'ON' CPAP for $6 \mathrm{~h}$ or until the 'stability criteria' was met.

- Method 3 (M3): CPAP was weaned 'OFF' in a similar way to 'M2', but the premature infants in this group were given humidified (water bubbler) nasal cannula (2 $\mathrm{mm}$ ) oxygen/ air (using an oxygen blender) during their time 'OFF' with a flow rate of $0.51 / \mathrm{min}$. This flow rate of $0.5 \mathrm{l} / \mathrm{min}$ with a $2 \mathrm{~mm}$ nasal cannula was adequate to be used with the water bubbler for humidification but did not generate a CPAP pressure. ${ }^{20}$ When $\mathrm{M} 3$ infants came off CPAP, they were continued on the nasal cannula for a further $24 \mathrm{~h}$ prior to complete removal of support.

The premature infant completed their method by successfully trialling OFF respiratory support for a period of 5 days. The centralised monitoring team reviewed the data for quality and completeness.

\section{STUDY NUMBERS AND STATISTICS}

Sample size calculations based on data (time to wean off CPAP) from 1997-2004 extracted from one of the neonatal units in the trial indicated that to reduce the time spent on CPAP by $15 \%$, a total sample size of 195 premature infants was required (65 in each group) to achieve $80 \%$ power and significance levels $(\alpha)$ of 0.05 or less.

A planned interim analysis was to be undertaken after 3 years. As a result of the interim analysis, the data-monitoring committee stopped the study. The DMC reviewed the interim analysis and concluded that the results clearly demonstrated effect sizes and within-group variations that were not going to vary from the interim analysis if recruitment was to continue. As a result, the study was stopped 1 year early.

Analyses of the data were performed on (1) all premature infants randomised to the trial (intention to treat (primary analysis)) first by analysing the data with adjustment for gender differences and Apgar scores and (2) on premature infants who completed the trial (per protocol (did not violate study protocol)). Those premature infants who were excluded in the per protocol analysis were those who (1) consultant withdrawal, (2) parental withdrawal, (3) transferred to other hospital or surgery, (4) died after randomisation. In addition to descriptive analyses, multivariate analyses were performed using generalised linear models adjusting for gender and Apgar scores at 1 and $5 \mathrm{~min}$. The models used linear scale response or Tweedie with log link, depending on the outcome measure's distribution. Bonferroni post-hoc pair-wise comparisons were carried out to adjust for repeated testing. For the analysis on the number of times taken to come off CPAP in M1; Mann-Whitney U tests were used to test the significance of gender and Spearman rho correlations were used to examine the effect of GA. Analyses were performed in SPSS version 17.0.

\section{RESULTS}

A total of 412 premature infants $<30$ weeks GA were identified during the recruitment period, 177 premature infants were enrolled in the study and were randomised as shown in figure 3 and table 1 . Of the 177 enrolled, 154 premature infants completed the trial (figure 3). The numbers in the M2 study group were slightly larger (table 1 ) as a result of the initial random selection process not using permuted blocks but simple randomisation (sealed envelopes done 
Table 2 Outcome indictors by CPAP weaning method (intension to treat multivariate analysis $\mathrm{n}=177$ )

\begin{tabular}{lllll}
\hline & M1 $(n=56)$ & $M 2(n=69)$ & $M 3(n=52)$ & Sig \\
\hline Time of wean ${ }^{\ddagger}$ & $11.3 \pm 0.8$ & $16.8 \pm 1.0^{*}$ & $19.4 \pm 1.3^{*}$ & $p<0.0001$ \\
Total days CPAP & $24.4 \pm 0.1$ & $38.6 \pm 0.1^{*}$ & $30.5 \pm 0.1^{*}$ & $p<0.0001$ \\
CGA OFF CPAP & $31.9 \pm 0.1$ & $34.1 \pm 0.1^{*}$ & $32.8 \pm 0.2^{*}$ & $p<0.0001$ \\
Oxygen duration $\neq$ & $24.1 \pm 1.5$ & $45.8 \pm 2.2^{*}$ & $34.1 \pm 2.0^{*}$ & $p<0.0001$ \\
BPD & $7 / 56(12.5 \%)$ & $29 / 69(42 \%) \dagger$ & $10 / 52(19 \%)$ & $p=0.011$ \\
Length of Admission\$ & $58.5 \pm 0.1$ & $73.8 \pm 0.1^{*}$ & $69.5 \pm 0.1^{*}$ & $p<0.0001$ \\
CGA at D/C\# & $35.8 \pm 0.1$ & $36.9 \pm 0.1^{*}$ & $36.9 \pm 0.1^{*}$ & $p<0.0001$ \\
\hline
\end{tabular}

Adjusted for gender and Apgar scores at 1 and 5 min within a generalised linear model.

${ }^{*}$ Significantly longer/more than $\mathrm{M} 1 \mathrm{p}<0.001,+\mathrm{p}<0.01$ (Bonferroni adjusted).

Estimated marginal means $\pm 1 S E, \ddagger$ days, $\$$ days in level 3 intensive care and \#D/C, discharge.

CGA, corrected GA; CPAP, continuous positive airway pressure.

outside of DiscoverQuick) and the stopping of the study before DiscoverQuick could achieve a balance. There were no significant differences in GA or birthweight (Bwt), although there was a significant difference in gender with a higher proportion of females in M1 (table $1, \mathrm{p}<0.01)$; and a significant difference in Apgar scores, with the premature infants in M3 having significantly higher scores at 1 and 5 min (table 1). There were no significant differences in corrected GA or current Wt between the groups at the time of randomisation and commencement of weaning (table 1).

The intension to treat analyses of the outcome indicators after adjusting for gender and Apgar scores are shown in table 2. Based on primary outcomes, time to wean CPAP and CPAP duration were significantly reduced with $\mathrm{M} 1$. Based on secondary outcomes, oxygen duration and incidence of BPD and length of admission were significantly shorter using M1 compared to the other methods.

Analysis as per protocol on the 154 premature infants who completed the trial found similar results (results not displayed). On reviewing data for premature infants in $\mathrm{M} 1$ who completed the trial per protocol $32 \%(16 / 50)$ came off CPAP on the first attempt, $42 \%$ (21/50) required $2-3$ attempts to come off and the remaining 28\% (14/50) took 4-13 attempts to come off CPAP. Males tended to take more attempts to come off CPAP than females (median attempts to come off CPAP $=3: 2$ / $M: F$, this was not significant). As expected, the more immature premature infants took significantly more attempts to come off than the more mature premature infants (median attempts to come off CPAP: 24/25 weeks=3, 26 weeks=3, 27 weeks=3.5, 28 weeks $=2$ and 29 weeks $=1$ attempts $\mathrm{p}<0.001)$. Babies more mature at 28-29 weeks GA took an average of $2.4 \pm 1.9$ or median of 2 attempts to come off CPAP, while the more immature $<28$ weeks GA took an average of $3.9 \pm 2.9$ or a median of three attempts.

\section{DISCUSSION}

In this RCT on CPAP weaning consideration was made of both when and how to wean premature infants OFF CPAP. Trial setup included construction of the trial 'stability criteria' and 'failure criteria': that is when to take premature infants OFF CPAP and when to put the babies back ON CPAP. The trial analysis included comparison of three methods of weaning premature infants OFF CPAP. No major critical incidents were recorded during the RCT although one infant in M1, two infants in M2 and one infant in M3 died from causes not related to the trial.

A previous study demonstrated that in infants $<34$ weeks GA who had their CPAP discontinued for $6 \mathrm{~h}$ have an increased oxygen requirement, increased respiratory rate and increased number of apnoea and bradycardias but take less time to come off CPAP. ${ }^{15}$ This study did not actually assess CPAP weaning methods but described the events when coming off CPAP and is consistent with M1 in the current trial. ${ }^{15}$ Two trials comparing weaning methods from a flow driver have shown that weaning from the initial CPAP pressures are better achieved by gradually decreasing the CPAP pressure rather than by slowly cycling the time off CPAP at that initial pressure. ${ }^{16} 17$ Soe et al found that in the more immature infants 24-27 weeks GA there was a trend towards less hospital days and less oxygen dependency at 36 weeks in the pressure reduction arm while there was no difference between the groups in the more mature infants 28-31 weeks GA. ${ }^{17}$ The confounding problem with both these trials is that the babies were taken off the CPAP at initial CPAP pressures. It is thus possible that the babies' respiratory system was still immature and unstable when taken off CPAP or the pressures were too high to be taken off. ${ }^{16}$ 17 Not withstanding these differences, in the current study, it has also been demonstrated that cycling is not necessary when the infant is taken off CPAP once reaching the stability criteria.

A recent trial of weaning CPAP in infants $>27$ weeks GA has shown that replacing the CPAP with nasal cannula at a flow of $21 / \mathrm{min}$ increases the days of oxygen, increases the duration of respiratory support but causes no difference regarding success of weaning. ${ }^{18}$ Singh et al also used low flow nasal cannulae during times off CPAP but this use was not shown to improve the time to wean and is similar to the current study. ${ }^{16}$ Low-flow and highflow nasal cannulae are now being used more frequently for both weaning and as a replacement for nasal CPAP. ${ }^{18} 20-24$ More research is needed with these alternative forms of respiratory support.

It remains a contentious issue as to when to wean premature infants off CPAP and when to return them to CPAP. Literature acknowledges the Columbia method and many units continue to only wean CPAP once the infant is breathing air. ${ }^{5} 2122$ Some units also include parameters such as weight or corrected GA before considering weaning. ${ }^{22}$ Thus knowing when to discontinue CPAP and when to replace CPAP has been poorly defined with most using an 'ad hoc' method. ${ }^{12} 13$ This study has utilised formulated 'stability' and 'failure' criteria based on previous studies and literature. ${ }^{5}$ 7121322 Others have used similar stability criteria to the current study but with higher oxygen requirements of $<30 \%$ oxygen. ${ }^{18}$ While the currently used 'stability' criteria in this trial may have been adequate for the more mature infants as they required only $2.4 \pm 1.9$ attempts to come off CPAP it may not be so for the more immature $<28$ weeks GA as they required an average of $3.9 \pm 2.9$ attempts. Having a 'stability' criterion (guidelines) in itself may be superior to using the 'ad hoc' method. Similarly having a 'failure' criterion and knowing when to put the infants back on CPAP may not have been adequate for the more immature infants and this may need to be modified in future trials. Despite the range of attempts to come 'OFF' CPAP, this RCT showed significant reductions in secondary outcome such as oxygen duration and BPD using M1.

Previous studies have found in infants $<30$ weeks GA an incidence of BPD of $15 \%$ in a hospital based population ${ }^{25}$ and an incidence of $26.9 \%$ for a multicentre cohort. ${ }^{26}$ While for the more 
immature infants $<28$ weeks GA an incidence varies from 17-69\% depending on the early pulmonary dysfunction and/ or infection. ${ }^{27-30}$ In those $<29$ weeks, the incidence is somewhat similar at 29-35\%. ${ }^{31}$ Although not powered specifically to study BPD, this study has shown that infants $<30$ weeks GA using M1 for weaning CPAP have a $12.5 \%$ incidence of BPD (table 2) with an oxygen duration of around 24 days compared to an oxygen duration of 46 and 34 days in the other methods, respectively (table 2). A possible mechanism is that $48 \mathrm{~h}$ on CPAP, once failed, has resulted in or allowed for full recruitment of the lungs and thus ensured the stability of the airways. In premature infants weaned by cycling off and on CPAP (M2) in short periods without oxygen/air support via nasal cannula, their lungs may not be fully recruited following the short period on CPAP and they may have partial atelectasis when the CPAP is removed. The cycle of recruitment and atelectasis may lead to airway damage and hence more BPD. In $\mathrm{M} 3$ where the infant was supported by low flow during times off, there was also a reduction in BPD although not time in oxygen and this requires further investigations.

Length of admission in the Neonatal unit and discharge to either home or to level 2 (special care units) was also significantly reduced in $\mathrm{M} 1$. This may have major health benefits and reduced health costs not only in the short term but also on a long-term basis. Premature infants especially with BPD are known to have long-term problems including home oxygen and further long term issues such as reactive airways and increased asthma symptoms with more hospitalisations. ${ }^{32}$ Follow-up is essential as there have been very little data addressing their respiratory and neurodevelopmental outcomes. ${ }^{4}$

In conclusion, this RCT on CPAP weaning has shown that in conjunction with the study stability and failure criteria, using M1 for weaning OFF CPAP significantly reduces time to wean CPAP, CPAP duration, oxygen duration, BPD and length of admission. Weaning CPAP using M1 had an impact on the intensity, costs and length of neonatal intensive care and may improve the long-term outcome in these small preterm infants. It is acknowledged that further trials are necessary to confirm these results.

Contributors Dr David Todd conceived the idea of the RCT on weaning preterm infants from CPAP. Other members of the group recruited patients, collected and input data. Dr Bruce Shadbolt set up the DiscoverQuick database, randomisation process and performed the statistical analysis.
Acknowledgements Theauthorsthankalltheclinicians who have helped in this study especially those who have aided in recruiting and data collection. Specifically, the authorsthankDrSrinivas Ramakrishnan, DrChetanPandit, Ms Kelly Cameron and Mrs Jane Baird. The authors also thank the independent 'data monitoring committee (DMC)' consisting of Ass Prof Graham Reynolds (Chair), Prof William Tarnow Mordi, Ass Prof Abdel-Latif Mohamed, Dr Tim McDonald and Mrs Jeni Williams. The terms of reference for the DMC were set up in accordance with the DAMOCLES Study Group for clinical trial monitoring. ${ }^{33}$

Funding Funding for the CPAP weaning study was obtained from (1) Canberra Hospital, Private Practice Fund, (2) Canberra Hospital, Newborn Intensive Care Foundation and (3) Fisher and Paykel Healthcare. Funding from the 3 sources was for Research Nurse Salary, Construction and Maintenance of Treonic Database, Postage, Some travel to present results at meetings. There was no involvement in the mechanics of the study by any of the funding sources.

\section{Competing interests None.}

Provenance and peer review Not commissioned externally peer reviewed.

Received 2 May 2011

Accepted 1 April 2012

Published Online First 18 May 2012

Arch Dis Child Fetal Neonatal Ed 2012;97:F236-F240. doi:10.1136/adc.2011-300133

\section{REFERENCES}

1. Gregory GA, Kitterman JA, Phibbs RH, et al. Treatment of the idiopathic respiratory-distress syndrome with continuous positive airway pressure. N Engl J Med 1971:284:1333-40.

2. John E, Thomas DB, Burnard ED. Influence of early introduction of continuous positive pressure breathing on the course of hyaline membrane disease. Aust Paediatr J 1976:12:276-80.

3. Engelke SC, Roloff DW, Kuhns LR. Postextubation nasal continuous positive airway pressure. A prospective controlled study. Am J Dis Child 1982;136:359-61

4. Gregory GA. Historical perspectives: Continuous Positive Airway Pressure (CPAP). Neo Reviews 2004:5:e1-e4

5. Polin RA, Sahni R. Newer experience with CPAP Semin Neonato/ 2002;7:379-89.

6. Sherman TI, Blackson T, Touch SM, et al. Physiologic effects of CPAP: application and monitoring. Neonatal Netw 2003:22:7-16.

7. De Paoli AG, Morley C, Davies PG. Nasal CPAP for neonates: what do we know in 2003. Arch Dis Child Fetal Neonatal ed 2003;88:F168-72.

8. De Paoli AG, Davis PG, Faber B, et al. Devices and pressure sources for administration of nasal continuous positive airway pressure (NCPAP) in preterm neonates. Cochrane Database Syst Rev 2008:1:CD002977.

9. Davis PG, Henderson-Smart DJ. Nasal continuous positive airways pressure immediately after extubation for preventing morbidity in preterm infants. Cochrane Database Syst Rev 2003;3:CD000143.

10. Davis PG, Janov R, Doyle L, et al. Randomized, controlled trial of nasal continuous positive airway pressure in extubation of babies weighing 600 to 1250 grams. Arch Dis Child Fetal Neonatal ed 1998:79:F54-7.

11. Greenough A, Prendergast M. Difficult extubation in low birthweight infants. Arch Dis Child Fetal Neonatal Ed 2008:93:F242-5.

12. Bowe L, Clarke P. Current use of nasal continuous positive airway pressure in neonates. Letter to editor Arch Dis. Child Fetal Neonatal ed 2005:90:F92-3.
13. Jardine L, Davies MW. Withdrawal of neonatal continuous positive airway pressure: current practice in Australia. Pediatr Int 2008;50:572-5

14. Bohlin K, Jonsson B, Gustafsson AS, et al. Continuous positive airway pressure and surfactant. Neonatology 2008;93:309-15.

15. Abdel-Hady H, Mohareb S, Khashaba M, et al. Randomized controlled trial of disconnection of nasal CPAP in stable preterm infants breathing room air. Act Paeditr 1998;87:82-7.

16. Singh S, Bowe L, Clarke P, et al. Is decreasing pressure or increasing time off the better strategy in weaning VLBW infants from nasal CPAP (abstract)? Euro J Pediatrics 2006;165:48.

17. Soe A. Weaning from nasal CPAP in premature infants. Inspire 2007:5:8-10.

18. Abdel-Hady H, Shouman B, Aly H. Early weaning from CPAP to high flow nasal cannula in preterm infants is associated with prolonged oxygen requirement: a randomized controlled trial. Early Hum Dev 2011;87:205-8.

19. DiscoverQuick. www.DiscoverQuick.com 2007-11.

20. Locke RG, Wolfson MR, Shaffer TH, et al. Inadvertent administration of positive enddistending pressure during nasal cannula flow. Pediatrics 1993;91:135-8.

21. Avery ME, Tooley WH, Keller JB, et al. Is chronic lung disease in low birth weight infants preventable? A survey of eight centers. Pediatrics 1987;79:26-30.

22. Rajiv PK. CPAP (continuous positive airway pressure): Bedside application in newborn, 2011. Second edition. Jaypee Brothers Medical Publishers Pty.

23. Sreenan C, Lemke RP, Hudson-Mason A, et al. High-flow nasal cannulae in the management of apnea of prematurity: a comparison with conventional nasal continuous positive airway pressure. Pediatrics 2001;107:1081-3.

24. Courtney SE, Pyon KH, Saslow JG, et al. Lung recruitment and breathing pattern during variable versus continuous flow nasal continuous positive airway pressure in premature infants: an evaluation of three devices Pediatrics 2001:107:304-8.

25. Todd DA, Jana A, John E. Chronic oxygen dependency in infants born at 24-32 weeks' gestation: the role of antenatal and neonatal factors. J Paediatr Child Health 1997:33:402-7.

26. Egreteau L, Pauchard JY, Semama DS, et al. Chronic oxygen dependency in infants born at less than 32 weeks' gestation: incidence and risk factors. Pediatrics 2001;108:E26.

27. Hannaford $\mathbf{K}$, Todd DA, Jeffery $\mathrm{H}$, et al. Role of ureaplasma urealyticum in lung disease of prematurity. Arch Dis Child Fetal Neonatal Ed 1999;81:F162-7.

28. Askie LM, Henderson-Smart DJ, Jones RA Management of infants with chronic lung disease of prematurity in Australasia. Early Hum Dev 2005;81:135-42

29. Laughon M, Bose C, Allred EN, et al. Antecedents of chronic lung disease in preterm infants. Arch Dis Child Fetal Neonatal ed 2011:96:F114-20.

30. Finer NN, Carlo WA, Walsh MC, et al. Early CPAP versus surfactant in extremely preterm infants. $N$ Eng/ J Med 2010;362:1970-9.

31. Morley CJ, Davis PG, Doyle LW, et al. Nasal CPAP or intubation at birth for very preterm infants. $N$ Engl J Med 2008;358:700-8

32. Halvorsen T, Skadberg BT, Eide GE, et al. Characteristics of asthma and airway hyperresponsiveness after premature birth. Pediatr Allergy Immunol 2005;16:487-94.

33. Campbell MK for the DAMOCLES Study Group. A proposed charter for clinical trial data monitoring committees: helping them to do their job well. Lancet 2005:365:711-22. 